\title{
The Improved Interleaved Voltage Measurement Method for Series Connected
}

\section{Battery Packs}

\author{
Bing Xia ${ }^{1,2}$, Truong Nguyen ${ }^{2}$, Jufeng Yang ${ }^{1}$, and Chris $\mathrm{Mi}^{1}{ }^{1, *}$
}

Department of Electrical and Computer Engineering, San Diego State University, 5500 Campanile Drive, San Diego, CA 92182, USA

Department of Electrical and Computer Engineering, University of California San Diego, 9500 Gilman Drive, La Jolla, CA 92093, USA

*Corresponding Author. Tel.: +1 (619) 594 3741; fax: +1 (619) 5942654

Email address: bixia@eng.ucsd.edu (B. Xia),tqn001@eng.ucsd.edu (T. Nguyen), cmi@sdsu.edu (C. Mi), jufeng.yang@mail.sdsu.edu (J. Yang).

\begin{abstract}
This paper proposes an improved interleaved voltage measurement method for battery packs in electric vehicles, which can distinguish between the sensor fault and cell fault without hardware or software redundancy. The coprime constraint in the basic interleaved measurement method is revisited with a new proof, and a graphical interpretation is introduced to visualize the constraint. Based on that, an improved measurement topology is developed to remove the coprime constraint which enables broader application. Moreover, the hardware implementation of the improved method is discussed based on cost and circuit design. The associated improvement in noise performance is mathematically formulated, and the noise limit and trend of the interleaved measurement method are derived. Simulation results match the noise analysis and experiments validate the broader application of the improved method.
\end{abstract}

Keywords: lithium-ion batteries; battery management system; interleaved voltage measurement method; fault tolerance; sensors; fault detection and isolation. 


\section{Introduction}

Vehicle electrification has been identified to be an effective approach to coping with energy crisis and greenhouse effect. The electrification promotes electric vehicles and hybrid electric vehicles to achieve better overall powertrain efficiency and to reduce emissions [1]. The energy storage system is an essential component in the electrified vehicles, and the majority of the systems nowadays utilizes lithium-ion batteries, which possess high energy/power density and long service life [2]. The high energy/power property of the lithium-ion batteries improves the vehicle performance and extends the travel range, nevertheless, the appealing properties may result into unexpected severe fire hazards when the batteries are not treated properly [3-6].

To ensure safe operation in electrified vehicles, the battery management system (BMS) is incorporated into the lithium-ion battery systems [7]. The BMS monitors the voltage, current and temperature values of battery cells, estimates the states of the batteries, and actively maintains their safe operation conditions by fault detection and mitigation strategies [8-10]. Previous research has investigated that, among all the three measurements, the voltage is the most crucial for battery safety because of the prompt response and high sensitivity to major electric faults, including over charge, over discharge, external short circuit and internal short circuit $[11,12]$. Therefore, a reliable voltage measurement system is critical to identify the safety status of the lithium-ion battery packs.

The conventional voltage sensing system measures the voltage of each battery cell with one voltage sensor for each battery cell. The one-to-one correspondence guarantees that the voltage for every cell is monitored. Unfortunately, this voltage measurement approach is not fault-tolerant. In other words, the one-to-one mapping cannot distinguish between sensor malfunction and cell failure, when an abnormal reading is observed. However, the cost of the corresponding mitigation 
actions varies significantly. The sensor failure is a low level fault, and the electric vehicle can be switched to the "limp home" mode for future maintenance, whereas the short circuit failure on a cell is a high level fault, which requires immediate power interruption for protection purposes.

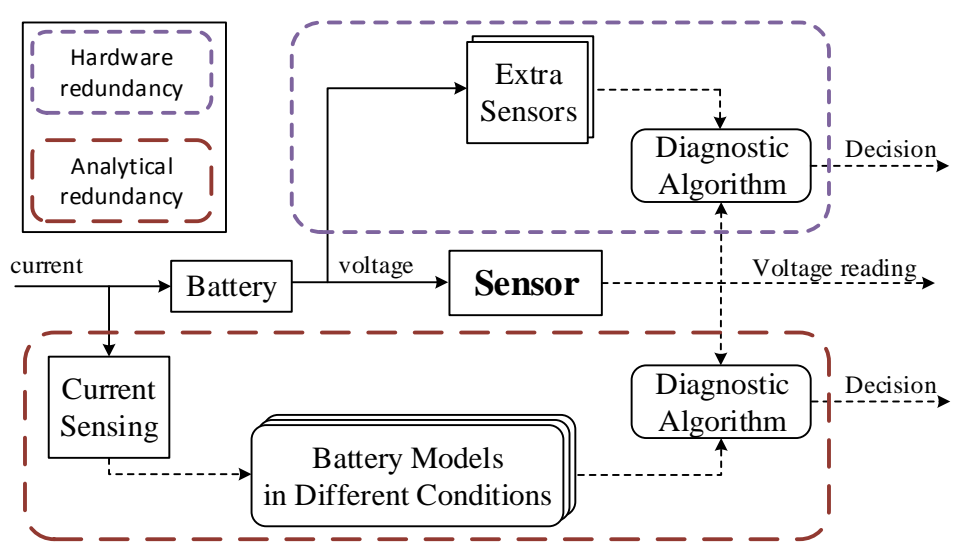

Fig. 1 Illustration of redundancy based fault detection and isolation.

Therefore, a sensor fault detection and isolation approach is necessary to enhance the reliability of voltage measurement in the battery packs. In general, the fault detection and isolation approaches utilize the concept of redundancy, which can be grouped into hardware redundancy and analytical redundancy [13], as illustrated in Fig. 1. The hardware redundancy compares the output signals of duplicative sensors to a same measurement. Usually, in order to determine the root cause of the fault, it requires at least two additional sensors to implement the majority vote algorithm. The inherent drawbacks of the hardware redundancy are that, 1) the system cost, size and complexity are increased; 2) the overall system reliability is lowered due to additional components [14]. The analytical redundancy saves the extra hardware expenses at the cost of the increase in computational burden. First, battery models in different working conditions, including normal and various fault modes, are required to be constructed and identified beforehand. Then the voltage outputs in different scenarios are estimated based on the current input. Finally, the sensor output is compared with model outputs to identify the fault [15-18]. However, the disadvantages of the software redundancy lie in that: 1) abundant preliminary work is required to 
build accurate battery models; 2) the robustness of the models cannot be guaranteed given the complex nonlinear behavior of batteries and disturbances in real applications; 2) when a fault is flagged, it can result from a sensor fault or a mismatched model.

Given the limitation of the redundancy based sensor fault detection methods, the authors proposed a fault-tolerant voltage measurement method for series connected battery packs, which does not require additional sensors or any effort in modeling [19]. For $n$ battery cells connecting in series, the proposed method requires $n$ voltage sensors. More importantly, each voltage sensor measures the voltage sum of $k$ battery cells $(2 \leq k<n)$ in an interleaved manner. In this way, each cell voltage is coupled with multiple voltage sensors. If any cell is in fault condition, the abrupt change in cell voltage will be captured by multiple voltage sensors. For example, the embodiment of $k=2$ is illustrated in Fig. 2(b). C2 is coupled with both V1 and V2. If C2 is decreased suddenly because of a fault occurrence, then a decrease with same amount should be observed in both V1 and V2. Since it is less possible for two voltage sensors to be in the same fault condition at the same time, a cell fault can be determined. Similarly, one sensor reading is also linked with multiple cell voltages in the inverse calculation. When a sensor fault occurs, the voltage of multiple cells will change abnormally in a specific pattern, and the sensor fault can be determined. Except the fault detection and isolation, the previous work also 1) discussed the feasibility of the method in real implementation; 2) demonstrated the associated noise level increase; 3) analyzed the effect of $k$ on the confidence level of cell/sensor fault detection and 4) derived that $n$ and $k$ should be coprime such that the measurement matrix is invertible, or the cell voltage can be calculated from the sensor readings, i.e., the sensor topology is valid. More detailed analysis and comparison with the existing measurement methods can be found in [19]. 
This manuscript is an extension of the previously proposed interleaved voltage measurement method. The contributions of this paper are: 1) a more concise derivation for the condition of valid sensor topology is provided; 2) based on the new derivation, an improved sensor topology is proposed which is not constrained by the coprime relation of $n$ and $k ; 3$ ) the associated noise level increase is mathematically formulated and the limit of the noise level is derived; (4) the improved sensor topology is proved to have a lower noise level than that of the previous work.

The paper first briefly introduces the key properties of the interleaved voltage measurement method. Then, a new proof for the condition of valid sensor topology is derived with the concept of the circulant matrix. A graphical interpretation is introduced to visualize the relation of $n$ and $k$ on the invertibility of the measurement matrix, based on which an improved sensor topology is developed. Next, the hardware implementation is discussed for the newly proposed sensor topology. After that, the noise level increase is mathematically formulated. The lower limit of the noise level is found, and the improved sensor topology is proved to have a lower noise level than that of the previously proposed topology. Finally, simulation results confirm the noise analysis and experiment results validate the feasibility of the improved interleaved measurement method.

\section{Brief review of the previous work}

Mathematically, the voltages of $n$ battery cells in series connection are correlated with the voltage readings from $n$ voltage sensors by (1).

$$
\mathbf{V}=\mathbf{A C}
$$

where $\mathbf{V}$ is an $n \times 1$ matrix that includes the voltage sensor readings, $\mathbf{C}$ is an $n \times 1$ matrix that includes the cell voltages, and $\mathbf{A}$ is an $n \times n$ matrix that characterizes the sensor topology. Conversely, cell voltage values can be calculated from the voltage sensor readings by

$$
\mathbf{C}=\mathbf{A}^{-1} \mathbf{V}=\mathbf{B V}
$$


where $\mathbf{B}$ is the inverse of the $\mathbf{A}$ matrix.

The conventional voltage sensor topology is illustrated in Fig. 2(a), whose A and $\mathbf{B}$ matrices are given in (3) and (4), respectively.

$$
\left[\begin{array}{c}
V_{1} \\
V_{2} \\
V_{3} \\
\vdots \\
V_{n}
\end{array}\right]=\left[\begin{array}{ccccc}
1 & 0 & 0 & \cdots & 0 \\
0 & 1 & 0 & & \vdots \\
0 & 0 & 1 & \ddots & \vdots \\
\vdots & & \ddots & \ddots & 0 \\
0 & \cdots & \cdots & 0 & 1
\end{array}\right]\left[\begin{array}{c}
C_{1} \\
C_{2} \\
C_{3} \\
\vdots \\
C_{n}
\end{array}\right]
$$
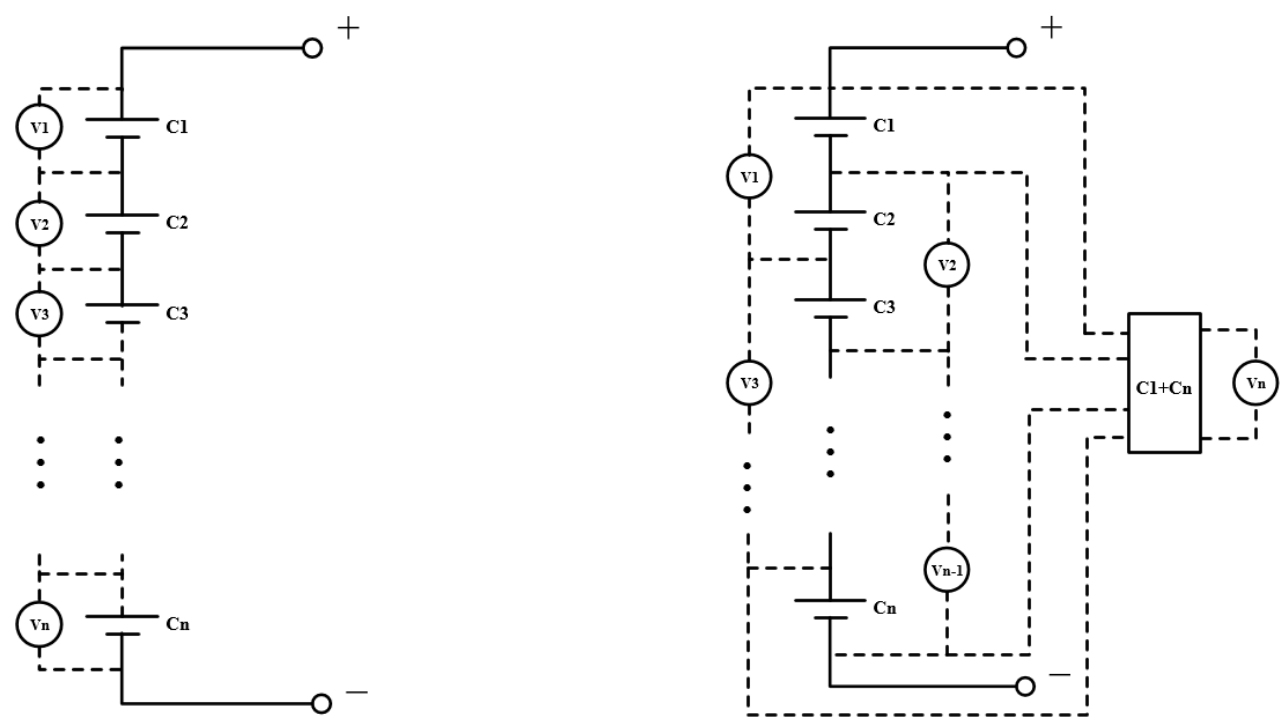

(a) The conventional measurement topology.

(b) The interleaved measurement topology.

Fig. 2 Schematics of two different measurement topologies.

Our previous work has introduced an interleaved voltage measurement topology that can distinguish between cell fault and sensor fault in series connected battery packs as shown in Fig. 2(b) [19]. The embodiment of $n=5$ for the interleaved topology is given in (5) and (6), respectively. It needs to be noted that the A matrix of the interleaved sensor topology is more "complex" than that of the conventional sensor topology, or the conventional sensor topology is a special case of the interleaved sensor topology where the sensor matrix is an identity matrix. 


$$
\left[\begin{array}{l}
V_{1} \\
V_{2} \\
V_{3} \\
V_{4} \\
V_{5}
\end{array}\right]=\left[\begin{array}{lllll}
1 & 1 & 0 & 0 & 0 \\
0 & 1 & 1 & 0 & 0 \\
0 & 0 & 1 & 1 & 0 \\
0 & 0 & 0 & 1 & 1 \\
1 & 0 & 0 & 0 & 1
\end{array}\right]\left[\begin{array}{l}
C_{1} \\
C_{2} \\
C_{3} \\
C_{4} \\
C_{5}
\end{array}\right]
$$

$$
\left[\begin{array}{l}
C_{1} \\
C_{2} \\
C_{3} \\
C_{4} \\
C_{5}
\end{array}\right]=\frac{1}{2}\left[\begin{array}{ccccc}
1 & -1 & 1 & -1 & 1 \\
1 & 1 & -1 & 1 & -1 \\
-1 & 1 & 1 & -1 & 1 \\
1 & -1 & 1 & 1 & -1 \\
-1 & 1 & -1 & 1 & 1
\end{array}\right]\left[\begin{array}{l}
V_{1} \\
V_{2} \\
V_{3} \\
V_{4} \\
V_{5}
\end{array}\right]
$$

From the fault diagnostic perspective, the identity measurement matrix is not favorable, because the one-to-one correspondence of the sensor to cell relation leaves the ambiguity when a fault occurs, i.e., there is no way to distinguish between sensor and cell failure when a voltage sensor shows an abnormal value. On the other hand, by observing the columns of $\mathbf{A}$ and $\mathbf{B}$ matrices of the interleaved topology, each cell's voltage value is associated with two voltage sensors as shown (5), and each sensor reading is conversely associated with multiple cell values based on a specific pattern as shown (6). It is important to note that these patterns are unique, otherwise the matrices will not have full column rank, which contradicts with the fact that $\mathbf{A}$ and $\mathbf{B}$ should be invertible. Hence, the unique patterns in both (5) and (6) can be utilized to isolate the source of abnormal signal changes, and thus determine the fault location.

In general, if the cell voltage is modeled as

$$
\mathbf{C}=\mathbf{C}_{\text {normal }}+\mathbf{C}_{\text {fault }}
$$

where $\mathbf{C}_{\text {normal }}$ is the cell voltages in normal conditions and $\mathbf{C}_{\text {fault }}$ is the cell voltage changes in fault conditions. Then $\mathbf{C}_{\text {fault }}$ can be isolated by

$$
\mathbf{C}_{\text {fault }}=\mathbf{C}-\mathbf{C}_{\text {normal }}=\mathbf{A}^{-1} \mathbf{V}-\mathbf{C}_{\text {normal }}
$$

Similarly, when sensor fault occurs, the sensor fault can be isolated by

$$
\mathbf{V}_{\text {faut }}=\mathbf{V}-\mathbf{V}_{\text {normal }}=\mathbf{V}-\mathbf{A C}_{\text {normal }}
$$

where $\mathbf{V}_{\text {normal }}$ is the voltage readings from sensors and $\mathbf{V}_{\text {faut }}$ is the voltage changes in readings in fault conditions. 
The measurement topology can be generalized to the case where the voltage sensors measure the sum of $k$ consecutive cell voltages $(2 \leq k<n)$, as indicated in (10).

$$
\left[\begin{array}{c}
V_{1} \\
V_{2} \\
\vdots \\
\vdots \\
V_{n-k+1} \\
V_{n-k+2} \\
\vdots \\
V_{n}
\end{array}\right]\left[\begin{array}{cccccccc}
1 & \cdots & \cdots & 1 & 0 & \cdots & \cdots & 0 \\
0 & 1 & \cdots & \cdots & 1 & 0 & \cdots & 0 \\
\vdots & \ddots & \ddots & & & \ddots & & \\
\vdots & & \ddots & \ddots & & & \ddots & \\
0 & \cdots & \cdots & 0 & 1 & \cdots & \cdots & 1 \\
1 & 0 & \cdots & \cdots & 0 & 1 & \cdots & 1 \\
\vdots & \ddots & \ddots & & & \ddots & \ddots & \vdots \\
1 & \cdots & 1 & 0 & \cdots & \cdots & 0 & 1
\end{array}\right]\left[\begin{array}{c}
C_{1} \\
C_{2} \\
\vdots \\
\vdots \\
C_{n-k+1} \\
C_{n-k+2} \\
\vdots \\
C_{n}
\end{array}\right](10) \quad\left[\begin{array}{c}
V_{1} \\
V_{2} \\
V_{3} \\
V_{4}
\end{array}\right]=\left[\begin{array}{cccc}
1 & 1 & 0 & 0 \\
0 & 1 & 1 & 0 \\
0 & 0 & 1 & 1 \\
1 & 0 & 0 & 1
\end{array}\right]\left[\begin{array}{c}
C_{1} \\
C_{2} \\
C_{3} \\
C_{4}
\end{array}\right]
$$

The analysis and derivation in the previous work lead to the following conclusions [19]:

a) The $n$ and $k$ need to be coprime, such that the resulting $\mathbf{A}$ matrix is invertible, otherwise the cell voltages cannot be obtained from the voltage readings. A simple example is given in (11), where the A matrix is not invertible when $n=4$ and $k=2$.

b) The larger the $k$ is, the more confidence level in the fault distinction, but the confidence level has negligible change when $k \geq 2$.

c) Given reasonable range of $k$ and $n$ in real applications, where $k \leq 4$ and $n \leq 16$, the noise level increases as $k$ or $n$ increases.

d) A larger $k$ requires higher voltage ranges for sensors and thus increases the cost.

Even though the previous work presents extensive discussion on the interleaved sensor topology, including fault isolation, detection confidence, hardware implementation, noise level and conditions for invertible A matrix in the form of (10), there are still a few problems that require continuing effort: 1) the derivation of the invertibility condition for $n$ and $k$ in (10) is too complicated; 2) the application of the A matrix in the form of (10) is constrained because $n$ and $k$ 
are required to be relative prime; 3) the limit and trend of the noise level variation need to be mathematically formulated.

\section{Extension of the measurement matrix}

\subsection{A new proof based on circulant matrix}

In this section, a new proof is presented for the invertibility condition of the A matrix in the form of (10). The new proof is not only more concise, but also sheds light on the performance improvement for the interleaved sensor topology, which is covered in later sections.

Proposition 1: A matrix is invertible if and only if its determinant is nonzero [20].

Combined with the following property of the determinant

$$
\operatorname{det}(\mathrm{A})=\prod_{i=0}^{n-1} \lambda_{i}
$$

where $\lambda_{i}$ is the $(i+1)^{\text {th }}$ eigenvalue of an $n \times n$ matrix, the next proposition is obtained.

Proposition 2: A matrix is invertible if and only if none of its eigenvalues is zero.

Now, we need to introduce the concept of the circulant matrix. A circulant matrix is a special kind of matrix, where each row is rotated one element to the right relative to the preceding row [21]. Indeed, the A matrix is a circulant matrix. The basic properties of the circulant matrix give the following proposition.

Proposition 3: The eigenvalues of the circulant matrix are the discrete Fourier transform (DFT) of its first row [22].

Denote the first row of an $n \times n$ circulant matrix as $\left[a_{0}, a_{1}, \cdots, a_{n-1}\right]$, then according to Proposition 3, the eigenvalues of (10) can be expressed as

$$
\lambda_{i}=\sum_{m=0}^{n-1} a_{m} e^{-j \frac{2 \pi}{n} i m}=\sum_{m=0}^{k-1} e^{-j \frac{2 \pi}{n} i m}, i=0,1, \cdots, n-1
$$


where $j$ is the unit imaginary number. Since the eigenvalues are sums of geometric series, their expressions can be simplified as

$$
\lambda_{i}=\left\{\begin{array}{cc}
k & i=0 \\
\frac{1-e^{-j \frac{2 \pi}{n} i k}}{1-e^{-j \frac{2 \pi}{n} i}} & i \neq 0
\end{array}\right.
$$

By Proposition 2, the invertibility of (10) is ensured when none of the eigenvalues in (14) is zero. Clearly, the eigenvalue is nonzero when $i=0$. When $i \neq 0$,

$$
\lambda_{i}=0 \Leftrightarrow 1-e^{-j \frac{2 \pi}{n} i k}=0 \Leftrightarrow e^{-j \frac{2 \pi}{n} i k}=1
$$

In order to keep all eigenvalues nonzero, it requires

$$
\frac{2 \pi}{n} i k \neq 2 p \pi \Leftrightarrow i k \neq p n \text {, for any } i=1,2, \cdots, n-1 \text { and any } p \in N
$$

The statement of (16) is equivalent to examine whether the multiple of $k$ is also a multiple of $n$. It shows that none of the $i k$ is a multiple of $n$ for $i=1,2, \cdots, n-1$, or none of them is a common multiple of $n$ and $k$. It is universally acknowledged that $n k$ is a common multiple of both $n$ and $k$, and when (16) is true, $n k$ is also the least common multiple of $n$ and $k$. This fact indicates $n$ and $k$ are coprime.

\subsection{Graphical interpretation of the choice of $n$ and $k$}

The first row of (10) consists of $k$ consecutive ' 1 's followed by $(n-k)$ consecutive ' 0 's. Its eigenvalues can be visualized by plotting the DFT of its first row versus the angle $2 \pi \frac{i}{n}, i=0,1, \cdots, n-1$ as (13). When $k$ is fixed and $n$ is a very large number, the profile of the eigenvalues can be obtained for this specific $k$. Then the eigenvalues of a small $n^{*}$ are the corresponding values at $2 \pi \frac{i}{n^{*}}, i=0,1, \cdots, n^{*}-1$ on the profile. 
As an example, the solid line in Fig. 3(a) shows the case of $n=100$ and $k=4$. The stems in Fig. 3(a) give the eigenvalues of $n=5$ and $k=4$. It can be seen that the eigenvalues of $n=5$ and $k=4$ are $(4,1,1,1,1)$. Since none of these eigenvalues is zero, the combination of $n=5$ and $k=4$ is invertible, and the corresponding measurement topology works.

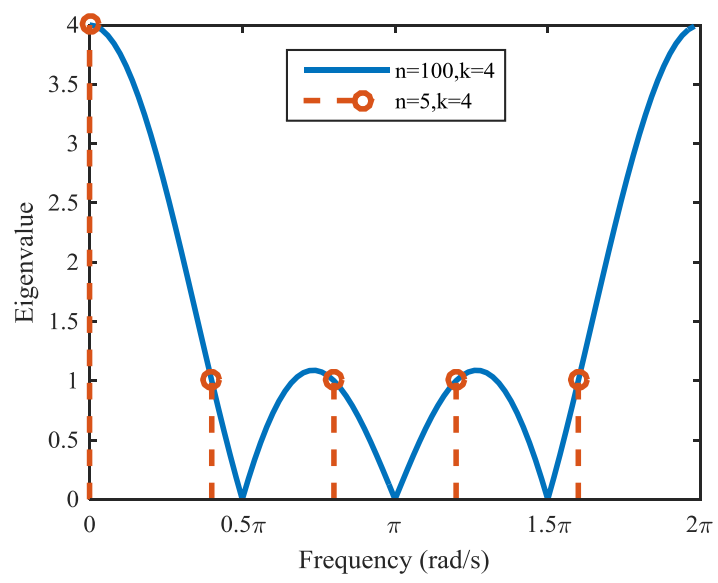

(a) DFT plot for $n=5$ and $k=4$.

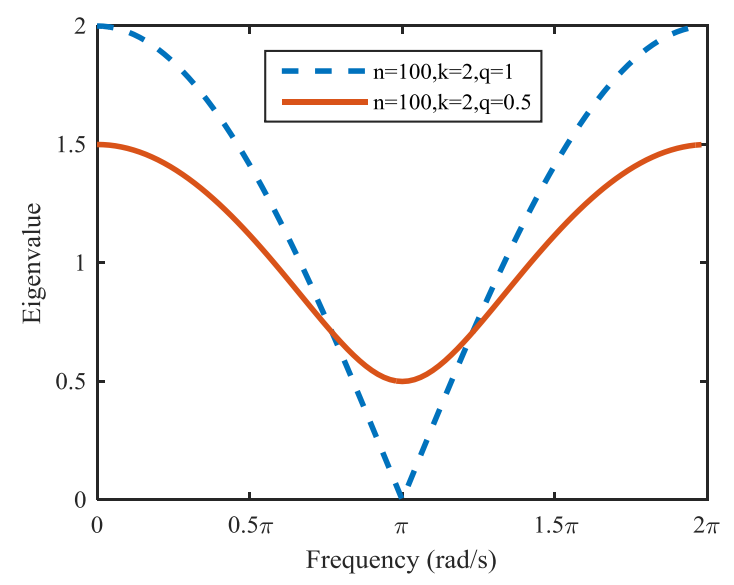

(b) Different DFT plot for $\mathrm{k}=2$.

Fig. 3 Graphical representation of eigenvalues of the measurement matrices.

It is interesting to note that there are three potential points on the profile, where the values are zero. If any of the stems falls on the zero points, it will lead to noninvertible measurement matrix and the corresponding measurement topology does not work. In general, the eigenvalue profile on the $2 \pi$ horizontal span is divided into $k$ parts by the $k-1$ evenly distributed zero points. If $n$ is a multiple of $k$, then there must exist an $i$ such that $\frac{i}{n}=\frac{1}{k}$, and this $i$ will place a stem at one of the zero points.

If the derivation from (14) to (16) is revisited, it is clear that the first eigenvalue will never be zero. As long as $k$ is not a factor of $n$, the stems will never fall on the zero points on the profile. The graphical interpretation matches the previous derivation and assists us to visualize the $n$ and $k$ choices. 


\subsection{Extension based on graphical interpretation}

Based on the mathematical analysis and the graphical interpretation, it proves that $n$ and $k$ need to be relative prime for the basic interleaved measurement matrix as (10). In real battery applications, the number of batteries in a module, or $n$, is usually a multiple of 6 or 12 . This is because these two numbers have many factors and it simplifies the pack design and assembly. Nevertheless, many of the factors are not favored in the measurement topology as shown in (10), because these factors cannot be used as $k$ to construct a valid measurement topology. For example, if the number of cells in a module is 12 , then the only choices for $k$ are 5, 7 and 11 . The high value of $k$ choices causes 1) more complicated hardware implementation; 2) higher voltage range for each sensor; 3) higher noise level compared with that of the traditional topology. In this section, an improved measurement matrix is proposed such that the constraint is removed.

The graphical interpretation shows that the limitation of $n$ and $k$ choices is caused by the zero points in the DFT profile. In other words, if the DFT profile is not zero anywhere over the $2 \pi$ span, then the corresponding A matrix should be always invertible.

As the case of two nonzero first row entries, the two entries can be normalized to be $a_{0}=1$, and $a_{1}=q(q>0)$. As an example, the DFT profiles of $q=1$ and $q=0.5$ are given in Fig. 3(b). It shows that, when $q=1$, which is the case of (10), the dashed profile equals to zero when the angle equals to $\pi$, whereas the solid line does not equal to zero anywhere over the horizontal span. This is because the zero eigenvalue emerges when

$$
\lambda_{i}=a_{0}+a_{1} e^{-j \frac{2 \pi}{n} i}=1+q e^{-j \frac{2 \pi}{n} i}=0, \text { for } i=0,1, \cdots, n-1
$$

In the case of $q=1, \lambda_{i}$ is zero when the angle is $\pi$, leaving the $\mathbf{A}$ matrix noninvertible. However, this situation can be easily improved by assigning different values for $a_{0}$ and $a_{1}$. 
Consequently, the DFT profile will never "touch" zero, and the resulting A matrix is always invertible no matter what $n$ value is chosen. Therefore, for the special case of two nonzero row entries, the A matrix is always invertible regardless of the choice of $n$ when $a_{0} / a_{1} \neq 1$.

Actually, this design can be extended to other number of nonzero entries as well. The ultimate goal is to ensure none of the eigenvalues is zero. In more general cases, the row entries in geometric series guarantee the invertibility of $\mathbf{A}$ for any combination of $n$ and $k$, as

$$
a_{i}=\left\{\begin{array}{cc}
q^{i} & i=0,1, \cdots, k-1, q>0 \text { and } q \neq 1 \\
0 & k \leq i \leq n-1
\end{array}\right.
$$

\section{Hardware implementation}
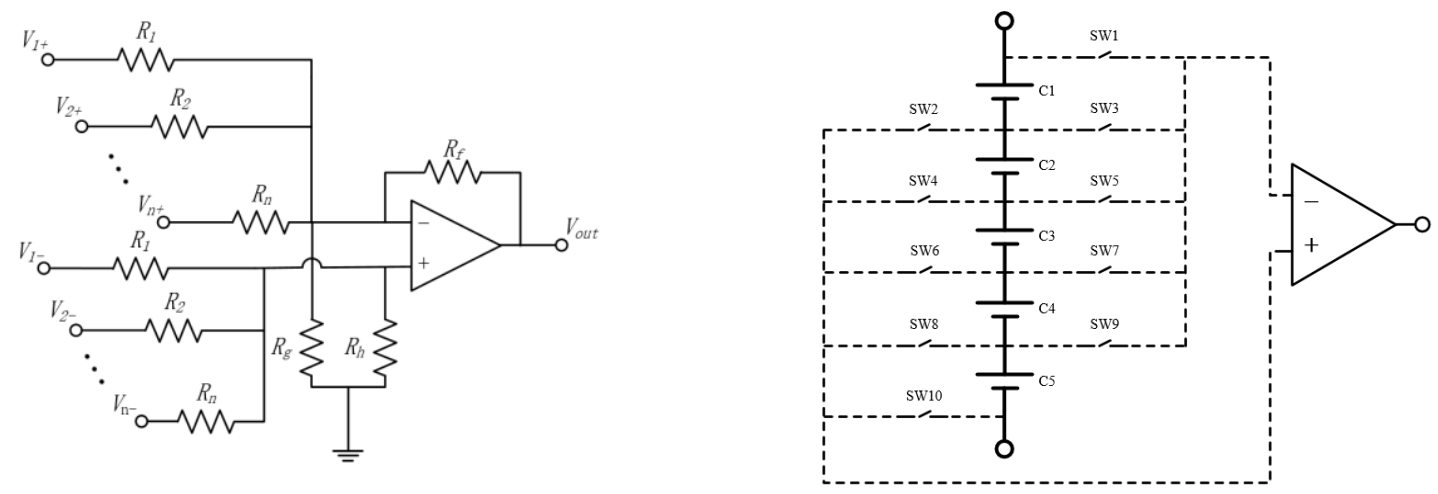

(a) Measurement circuit for voltage sum calculation of separate cells.

(b) The conventional measurement topology requires $2 n$ switches.
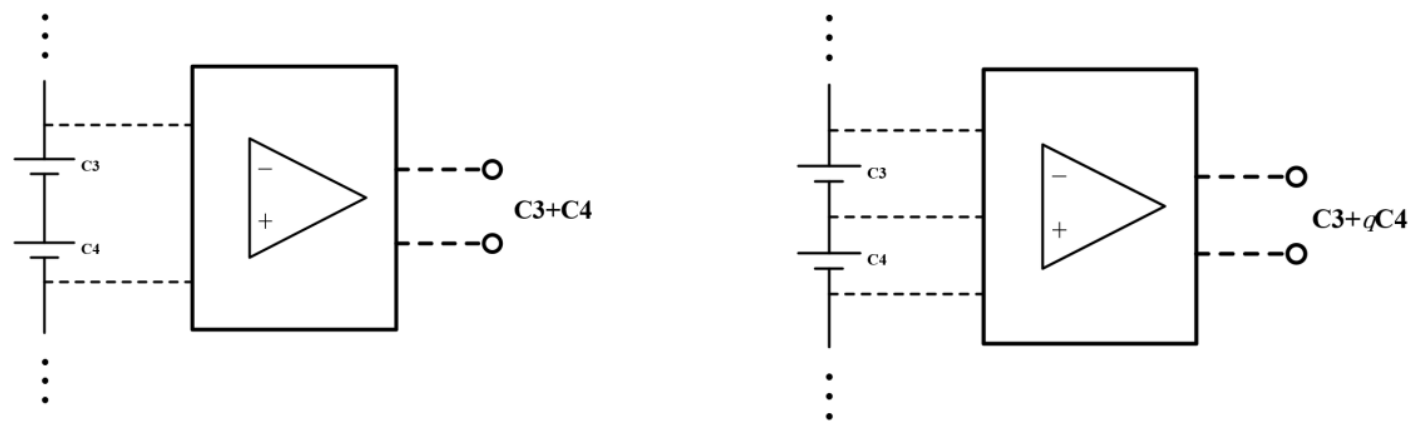

(c) Implementation for the basic interleaved topology

(d) Implementation for the improved interleaved topology

Fig. 4 Circuits for voltage measurements. 


\subsection{Voltage sum of separate cells}

The realization of the interleaved topology is limited to the hardware implementation. The first problem is that the voltage sum of cells is hard to obtain when they are separated by other cells, which is needed as the realization of the last row of (5). The previous work has proposed a circuit that calculates the separate voltage sum with additional pairs of resistors [19] compared with the classic differential amplifiers, as given in Fig. 4(a), where $V_{i+}$ and $V_{i-}$ connect to the positive and negative terminals of battery cells. With slight modification, it can be used to realize the case with different nonzero entries. For example, if there are only two separate cells, $V_{o}=-\left[\left(V_{1+}-V_{1-}\right)+q\left(V_{2+}-V_{2-}\right)\right]$ can be realized when the resistor values follows the relation as

$$
R_{1}=R_{f}, R_{2}=\frac{1}{q} R_{f}, R_{h}=\frac{R_{f} R_{g}}{R_{f}+R_{g}}
$$

\subsection{Number of switches}

The typical sequential voltage measurement circuit is given in Fig. 4(b) [23]. It shows that the conventional measurement topology requires $2 n$ switches because two terminals conduct to the measurement circuit at each sampling interval.

For the measurement topology of (10), a same number of switches are needed, because the consecutive cell measurements also need two terminals conducting to the measurement circuit within one sampling, as shown in Fig. 4(c). If the row entries of the A matrix are not the same, the number of switches needs to be increased due to an additional path to the measurement circuit within each sampling as shown in Fig. 4(d). Given different row entries, this additional path provides more information from the cell voltages, which is the key to make the measurement matrix invertible. In the general case of (18), where the first row entries are different and consecutive, the number of switches increases as $k$ increases. Thus, from the cost perspective, the choice of two nonzero entries is most favored in the interleaved voltage measurement topology.

\section{Noise analysis}




\subsection{General analysis of noise level gain}

The noise of the voltage sensors can be modeled as the white noise with a zero mean value and a standard deviation of $\sigma$. Here we assume that: 1) $\sigma$ is the noise standard deviation of the voltage sensor whose range covers a typical cell voltage; 2) the noise standard deviation is proportional to the number of series cells it measures; 3 ) the noises of different voltage sensors are uncorrelated. Then the variance of the sensor measurement can be expressed as

$$
\operatorname{Var}\left(V_{i}\right)=\sum_{p=0}^{n-1}\left(a_{p} \sigma\right)^{2}, i=0,1, \cdots, n-1
$$

The noise level gain, $G_{N L}$, is defined as the noise standard deviation of the cell voltages obtained from the interleaved topology divided by that of the conventional topology. With

Proposition 4, denote the row entries of the inverse of $\mathbf{A}$ matrix as $\left[b_{0}, b_{1}, \cdots, b_{n-1}\right]$, then the noise level gain can be derived as (21).

Proposition 4: The inverse of an invertible circulant matrix is also a circulant matrix [24].

$$
G_{N L}=\sqrt{\frac{\operatorname{Var}\left(\sum_{i=0}^{n-1} b_{i} V_{i}\right)}{\sigma^{2}}}=\sqrt{\frac{\operatorname{Var}\left(V_{i}\right) \sum_{i=0}^{n-1} b_{i}^{2}}{\sigma^{2}}}=\sqrt{\frac{\left(\sum_{i=0}^{n-1} a_{i} \sigma\right)^{2} \sum_{i=0}^{n-1} b_{i}^{2}}{\sigma^{2}}}=\sqrt{\left(\sum_{i=0}^{n-1} a_{i}\right)^{2} \sum_{i=0}^{n-1} b_{i}^{2}}
$$

It is discussed in [25] that the general solution of the inverse of a circulant matrix is given as

$$
b_{i}=\sum_{m=0}^{n-1} \frac{e^{j \frac{2 m i \pi}{n}}}{n\left(a_{0}+a_{1} e^{j \frac{2 m \pi}{n}}+\cdots+a_{n-2} e^{-j \frac{4 m \pi}{n}}+a_{n-1} e^{-j \frac{2 m \pi}{n}}\right)}, i=0,1, \cdots, n-1
$$

For simplicity, $b_{i}$ is expressed as an $n \times n$ matrix as (23). If the entry in the $(i+1)^{\text {th }}$ row and $(m+1)^{\text {th }}$ column is denoted as $\beta_{i, m}$, then when $i=0$, the numerators of $\beta_{0, m}, m=0,1, \ldots, n-1$, are 1 ; when $m=0$, the exponential terms in the denominators are 1 . Next, the matrix representation of $b$ is used to prove a proposition. 


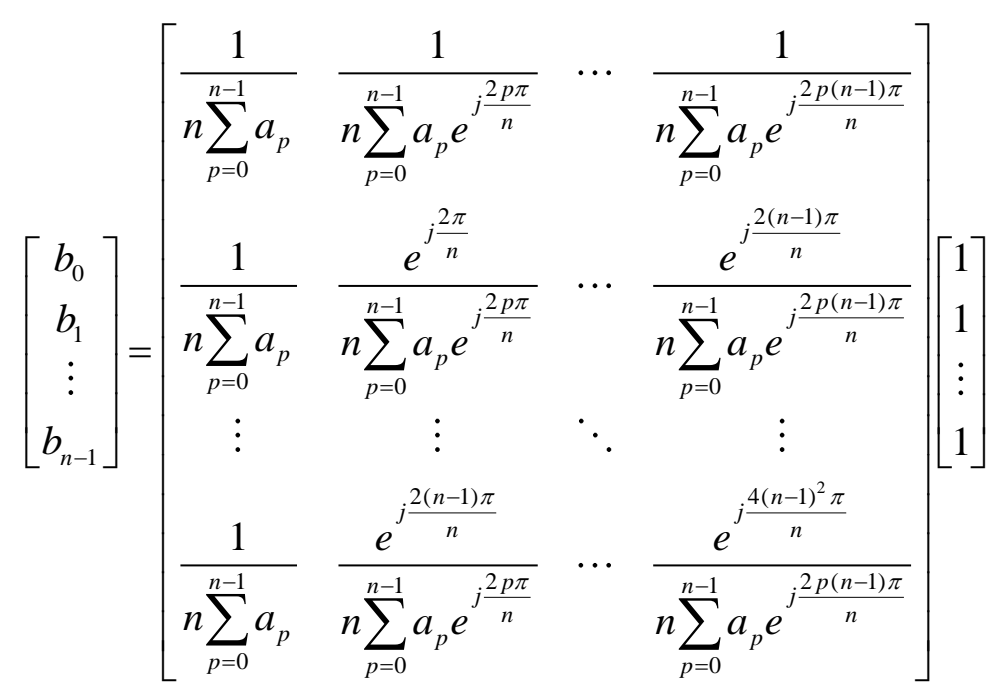

Proposition 5: The sum of the row entries of $\mathbf{B}$ matrix equals the inverse of the sum of the row entries of A matrix.

First, this proposition can be proved by assigning special values to cell voltages. When $\mathrm{C}_{\mathrm{i}}=1$, for $i=1,2, \ldots, n, V_{i}=\sum_{p=0}^{n-1} a_{p}$ as (24). As $\mathrm{C}_{\mathrm{i}}$ is calculated from $\mathbf{B}$ matrix as (25), the equation indicates $\sum_{p=0}^{n-1} a_{p} \sum_{p=0}^{n-1} b_{p}=1$

$$
\left[\begin{array}{c}
V_{1} \\
V_{2} \\
\vdots \\
V_{n}
\end{array}\right]=\left[\begin{array}{cccc}
a_{0} & a_{1} & \cdots & a_{n-1} \\
a_{n-1} & a_{0} & a_{1} & \cdots \\
\ddots & \ddots & \ddots & \ddots \\
a_{1} & \cdots & a_{n-1} & a_{0}
\end{array}\right]\left[\begin{array}{c}
1 \\
1 \\
\vdots \\
1
\end{array}\right]
$$

$$
\left[\begin{array}{c}
1 \\
1 \\
\vdots \\
1
\end{array}\right]=\left[\begin{array}{cccc}
b_{0} & b_{1} & \cdots & b_{n-1} \\
b_{n-1} & b_{0} & b_{1} & \cdots \\
\ddots & \ddots & \ddots & \ddots \\
b_{1} & \cdots & b_{n-1} & b_{0}
\end{array}\right]\left[\begin{array}{c}
V_{1} \\
V_{2} \\
\vdots \\
V_{n}
\end{array}\right]
$$

Proposition 5 can also be proved by (23) in a straightforward way. Except the first column, the sum for each column equals to 0 . The sum of $\mathbf{B}$ matrix then equals the sum of the first column and the result is $1 / \sum_{p=0}^{n-1} a_{p}$, which is the inverse of the sum of row entries of $\mathbf{A}$ matrix.

The same technique can be applied to calculate the sum of $b_{i}{ }^{2}$, which is an essential part of $G_{N L}$. An $n \times\left(n+C_{n}^{2}\right)$ matrix is constructed as (26), where $C_{n}^{2}$ is the number of combination sets for choosing 2 objects from $n$ distinct objects. 


$$
\left[\begin{array}{c}
b_{0}^{2} \\
b_{1}^{2} \\
\vdots \\
b_{n-1}^{2}
\end{array}\right]=\left[\begin{array}{cccccc}
\beta_{0,0}^{2} & \cdots & \beta_{0, n-1}^{2} & 2 \beta_{0, \delta_{0}(1)} \beta_{0, \delta_{0}(2)} & \cdots & 2 \beta_{0, \delta_{n-1}(1)} \beta_{0, \delta_{n-1}(2)} \\
\beta_{1,0}^{2} & \cdots & \beta_{1, n-1}^{2} & 2 \beta_{1, \delta_{0}(1)} \beta_{1, \delta_{0}(2)} & \cdots & 2 \beta_{1, \delta_{n-1}(1)} \beta_{1, \delta_{n-1}(2)} \\
\vdots & & \vdots & \vdots & & \vdots \\
\beta_{n-1,0}^{2} & \cdots & \beta_{n-1, n-1}^{2} & 2 \beta_{n-1, \delta_{0}(1)} \beta_{n-1, \delta_{0}(2)} & \cdots & 2 \beta_{n-1, \delta_{n-1}(1)} \beta_{n-1, \delta_{n-1}(2)}
\end{array}\right]\left[\begin{array}{c}
1 \\
\vdots \\
1
\end{array}\right]
$$

where $\delta_{i} \in S_{n}, S_{n}$ is the set of all combinations of choosing 2 values from $0,1, \cdots, n-1$, and $\delta_{i}$ is one of the combination within $S_{n}$.

It can be observed from the $n \times\left(n+C_{n}^{2}\right)$ matrix that every entry is closely related to the product of $\beta$. For a given column, its entries can be expressed as

$$
\alpha \beta_{i, u} \beta_{i, v}=\alpha \frac{e^{j \frac{2 i \pi}{n} u} e^{j \frac{2 i \pi}{n} v}}{n \sum_{p=0}^{n-1} a_{p} e^{j \frac{2 p u \pi}{n}} n \sum_{p=0}^{n-1} a_{p} e^{j \frac{2 p v \pi}{n}}}=\alpha \frac{e^{j \frac{2(u+v) \pi i}{n}}}{n^{2} \sum_{p=0}^{n-1} a_{p} e^{j \frac{2 p u \pi}{n}} \sum_{p=0}^{n-1} a_{p} e^{j \frac{2 p v \pi}{n}}}
$$

where $0 \leq u \leq v \leq n-1$, and $\alpha$ depends on the column index $m$ (note $m$ starts from 0 ) as

$$
\alpha= \begin{cases}1, & m=0,2, \cdots, n-1 \\ 2, & m=n, \cdots, n+C_{n}^{2}-1\end{cases}
$$

There are two important facts for (27): 1) the denominators are the same for the same column; 2) the common ratio of the numerators for the same column is $e^{j \frac{2(u+v) \pi}{n}}$. Thus, the sum of a column can be written as

$$
\alpha \sum_{i=0}^{n-1} \beta_{i, u} \beta_{i, v}=\left\{\begin{array}{cl}
\alpha \frac{1}{n \sum_{p=0}^{n-1} a_{p} e^{j \frac{2 p u \pi}{n}} \sum_{p=0}^{n-1} a_{p} e^{j \frac{2 p v \pi}{n}}} & , e^{j \frac{2(u+v) \pi}{n}}=1 \\
0 & , e^{j \frac{2(u+v) \pi}{n}} \neq 1
\end{array}\right.
$$

It shows that so long as the common ratio is not 1 , the sum of one column is 0 . For an odd number of $n$, there are two possible situations where the common ratio is 1: 1) when $m \leq n-1$, $u=v=0 ; 2)$ when $m>n-1, u+v=n$. Obviously, the number for case 1) is 1 , and the number for case 
$2)$ is $(n-1) / 2$. Combined with similar cases where $n$ is an even number, the Cauchy-Schwarz inequality in (30) leads to (31), (32) and finally Proposition 6.

$$
\begin{gathered}
\sum_{p=0}^{n-1} a_{p} e^{j p \theta} \sum_{p=0}^{n-1} a_{p} e^{j p(2 \pi-\theta)}=\sum_{p=0}^{n-1} a_{p} e^{j p \theta} \sum_{p=0}^{n-1} a_{p} e^{-j p \theta} \geq\left(\sum_{p=0}^{n-1} a_{p}\right)^{2} \\
\sum_{i=0}^{n-1} b_{i}^{2}=\frac{1}{n\left(\sum_{p=0}^{n-1} a_{p}\right)^{2}}+2 \frac{1}{n \sum_{p=0}^{n-1} a_{p} e^{j \frac{2 p \pi}{n}} \sum_{p=0}^{n-1} a_{p} e^{j \frac{2 p(n-1) \pi}{n}}}+\cdots \\
+2 \frac{1}{n \sum_{p=0}^{n-1} a_{p} e^{j \frac{2 p \pi}{n}\left(\frac{n}{2}-1\right)} \sum_{p=0}^{n-1} a_{p} e^{j \frac{2 p(n-1) \pi}{n}}\left(\frac{n}{2}+1\right)} \\
>\frac{1}{n\left(\sum_{p=0}^{n-1} a_{p}\right)^{2}}+2 \frac{1}{n\left(\sum_{p=0}^{n-1} a_{p}\right)^{2}}+\cdots+2 \frac{1}{n\left(\sum_{p=0}^{n-1} a_{p}\right)^{2}} \\
=\frac{1}{n\left(\sum_{p=0}^{n-1} a_{p}\right)^{2}}+2 \frac{n-1}{2} \frac{1}{n\left(\sum_{p=0}^{n-1} a_{p}\right)^{2}} \\
G_{N L}=\sqrt{\left(\sum_{i=1}^{n-1} a_{i}\right)^{2} \sum_{i=1}^{n} b_{i}^{2}}>1
\end{gathered}
$$

Proposition 6: The noise level for the interleaved measurement topology is always larger than that of the conventional topology.

\subsection{Noise level gains for two nonzero entries}

The first row of the general measurement matrix with two nonzero row entries is $[1, q, 0, \cdots, 0]$. The square of noise level gain can be expressed as

$$
G_{N L}^{2}=\left(\sum_{i=1}^{n} a_{i}\right)^{2} \sum_{i=1}^{n} b_{i}^{2}=\frac{\alpha}{n} \sum_{p=0}^{n-1} \frac{(1+q)^{2}}{\left(1+q e^{j p \theta_{p}}\right)\left(1+q e^{-j p \theta_{p}}\right)}
$$

where $\theta_{p}=\frac{2 p \pi}{n}$.

The derivative of the term is 


$$
\frac{d G_{N L}^{2}}{d q}=\frac{\alpha}{n} \sum_{p=0}^{n-1} \frac{\left(e^{j p \theta_{p}}+e^{-j p \theta_{p}}-2\right)\left(q^{2}-1\right)}{\left(q e^{-j p \theta_{p}}+1\right)^{2}\left(q e^{j p \theta_{p}}+1\right)^{2}}
$$

The two terms in the denominator are conjugate pairs, so their product is always positive. The first term in the numerator is always smaller than zero because the sum of the conjugate pairs are smaller than 2. Given the fact that $q$ and $G_{N L}$ are always positive, the following relation holds for the case of two nonzero entries

$$
\frac{d G_{N L}}{d q} \begin{cases}>0, & q \in(0,1) \\ =0, & q=1 \\ <0, & q \in(1,+\infty)\end{cases}
$$

Eq. (35) indicates that the global maximum of $G_{N L}$ is at $q=1$. The above analysis proves that, given two nonzero entries, the measurement noise level is the largest when $q=1$, and the measurement noise level can be decreased by varying the value of $q$.

\section{Simulation}

Simulations are set up in MATLAB to demonstrate the trend of the noise level gain. In the simulation, the measurement noise is white, and its standard deviation is linear with the range of the voltage sensors. The cell voltage values are then calculated from the contaminated measurements and the standard deviation of the calculated cell voltages is compared with that of the conventional sensor topology. The cell voltages are set to be $3 \mathrm{~V}, n$ ranges from 3 to 12 , and $q$ ranges from 0 to 5 at a step of 0.01 . The noise level gains are plotted in Fig. 5. 


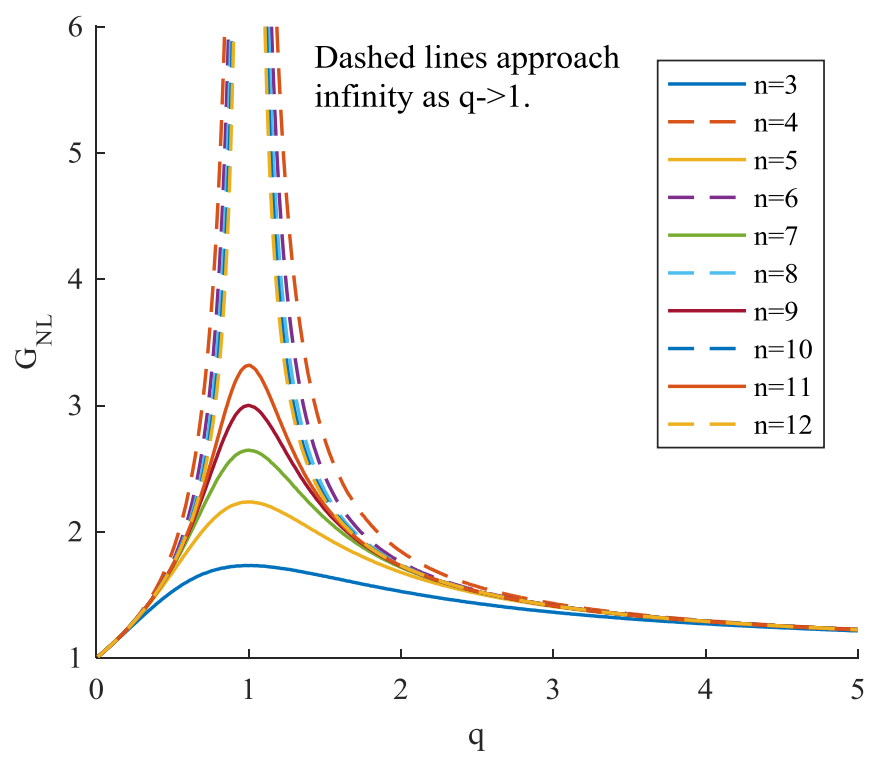

Fig. 5 Simulated $G_{N L}$ for different $q$ and different $n$.

Here list several important observations from Fig. 5:

a) The $G_{N L}$ converges to 1 as $q$ goes to 0 . This is because when $q$ equals to 0 , the measurement topology is equivalent to the conventional sensor topology.

b) The $G_{N L}$ converges to 1 as $q$ goes to infinity. An extension from Proposition 4 shows that the $G_{N L}$ does not change when all the row entries are multiplied by a nonzero number. Denote the measurement matrix with first row entries of $[1, q, 0, \cdots, 0]$ as $\operatorname{circ}(1, q, 0, \cdots, 0)$ . Then the $G_{N L}$ of $\operatorname{circ}(1, q, 0, \cdots, 0)$ is the same as that of $\operatorname{circ}\left(q^{-1}, 1,0, \cdots, 0\right)$, which is obviously the same as $\operatorname{circ}\left(1, q^{-1}, \mathrm{O}, \cdots, 0\right)$. As $q$ goes to infinity, the proposed topology converges to the conventional measurement topology and $G_{N L}$ converges to 1 .

c) The $G_{N L}$ is always greater than 1 except at $q=0$. This matches the derivation in Proposition 6.

d) When $n$ is an even number, the $G_{N L}$ goes to infinity at $q \rightarrow 1$, as illustrated by the dashed lines. When $q=1, n$ and $k$ should be relative prime to obtain invertible $\mathbf{A}$ matrices. 
However, the even numbers are not prime to $k$ in this case. Therefore, the measurement topology cannot be solved, and the $G_{N L}$ goes to infinity.

e) When $n$ is an odd number, the $G_{N L}$ is at its global maximum at $q=1$, as illustrated by the solid lines. This matches the derivation in Section 5.2.

\section{Experimental}

In order to demonstrate the broader application of the improved measurement topology, an experiment is conducted for the case of $n=6$ with two nonzero first row entries of 1 and 0.5 . It needs to be noticed that $n=6$ is not invertible in the previous work where both of the two row entries are 1. The schematic of the experimental setup is shown in Fig. 6(a), and the picture for the interleaved measurement circuit board is given in Fig. 6(b).

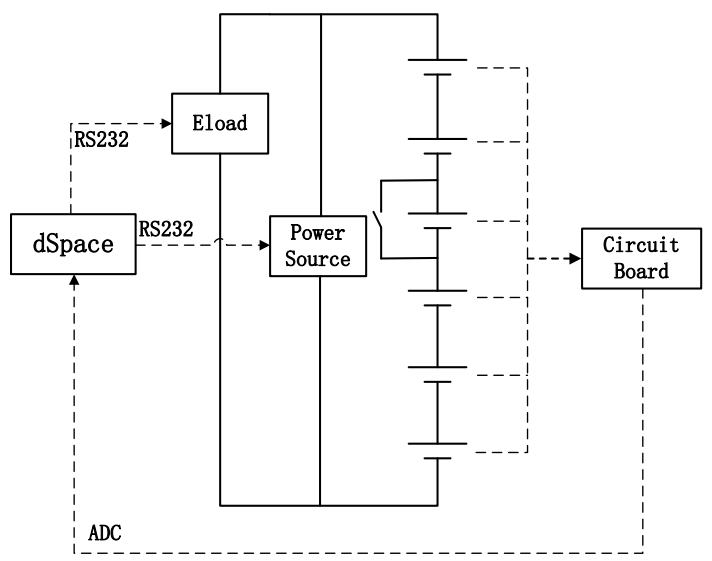

(a) Schematic of experiment setup.

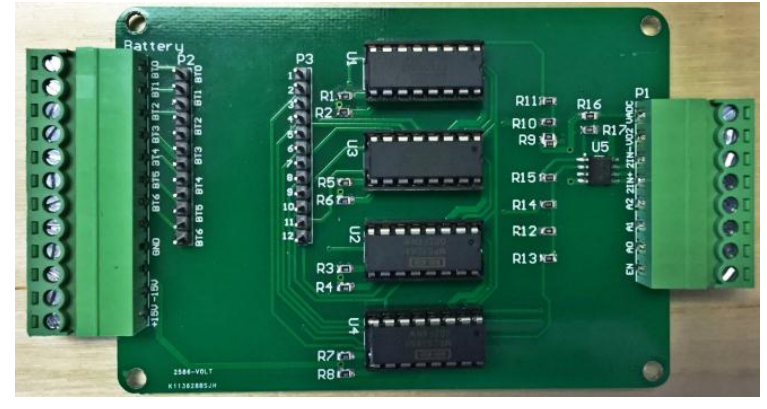

(b) Circuit board for validation.

Fig. 6 Experiment setup for validation.

The urban dynamometer driving schedule (UDDS) is applied to the six-cell string, and an external short circuit fault is induced at $200.7 \mathrm{~s}$ by shorting C3. The results of the cell fault experiment are given in Fig. 7. The sensor fault is induced at $173.4 \mathrm{~s}$ by disconnecting the jump wire in the sensing trace of V6. The results for the sensor fault experiment are given in Fig. 8. 

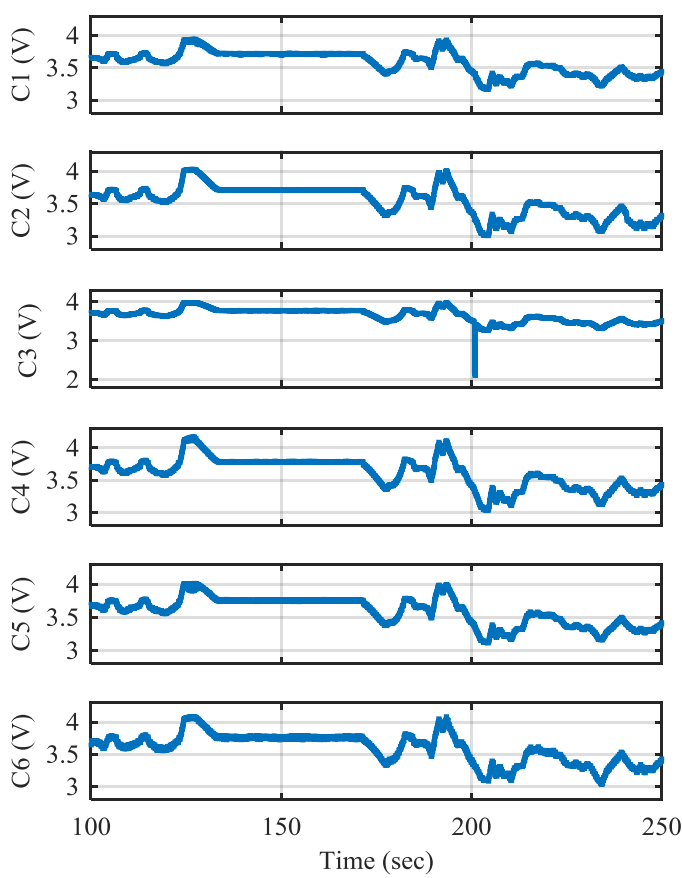

(a) Cell voltage values.
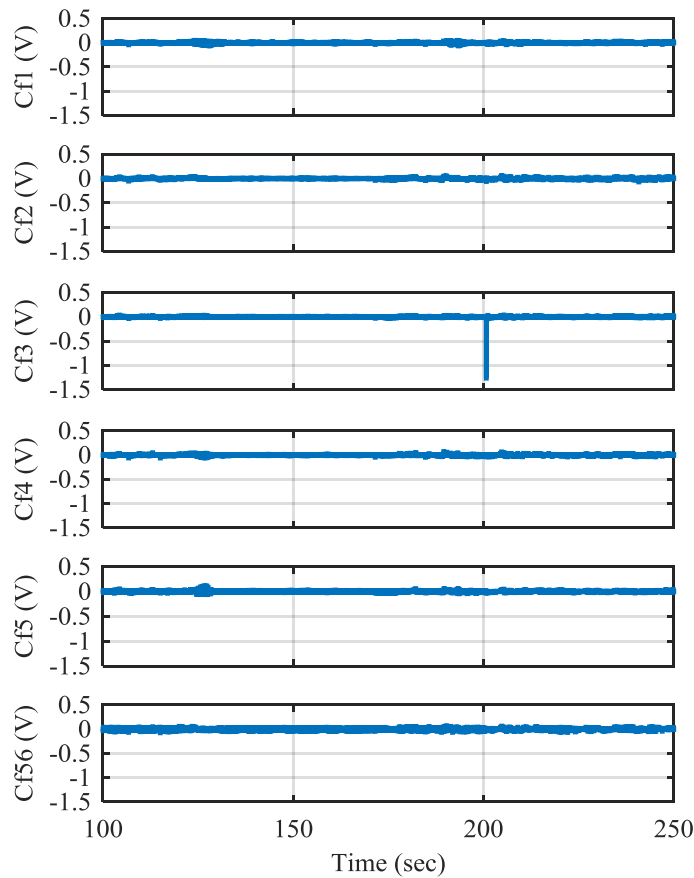

(c) Cell fault signal.
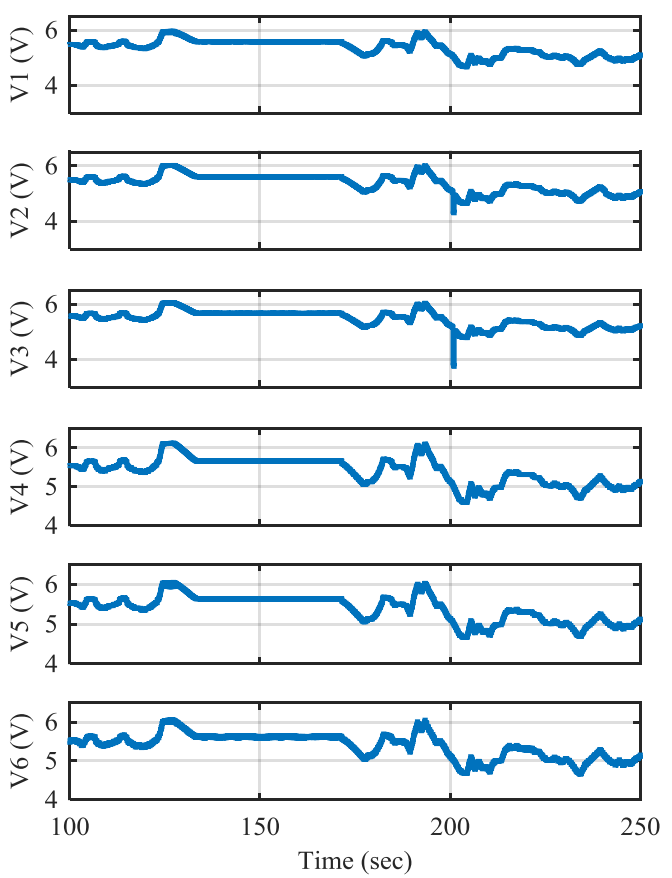

(b) Voltage measurements.
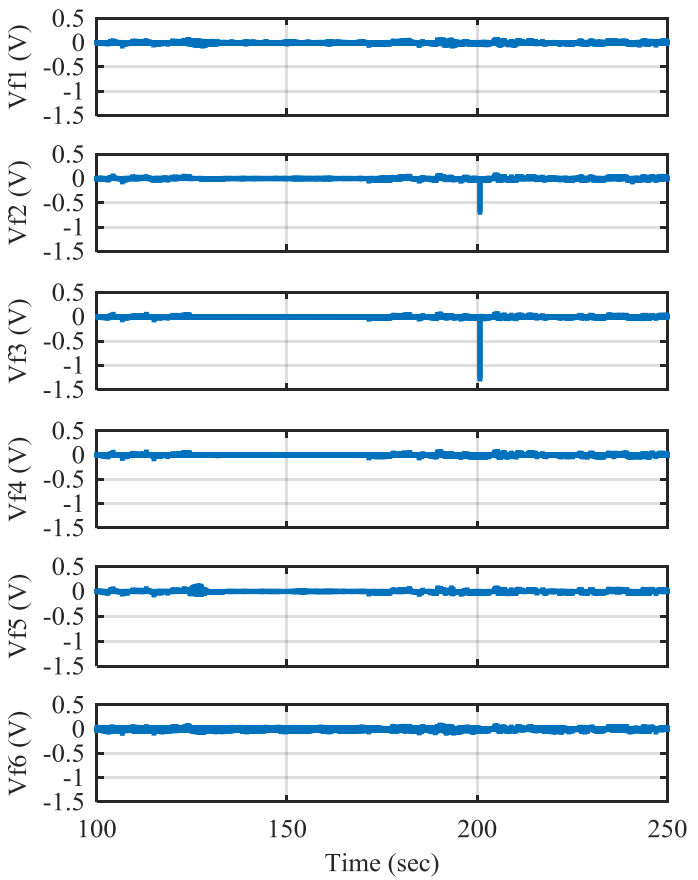

(d) Sensor fault signal.

Fig. 7 Experiment results for cell fault diagnostic at $200.7 \mathrm{~s}$. 

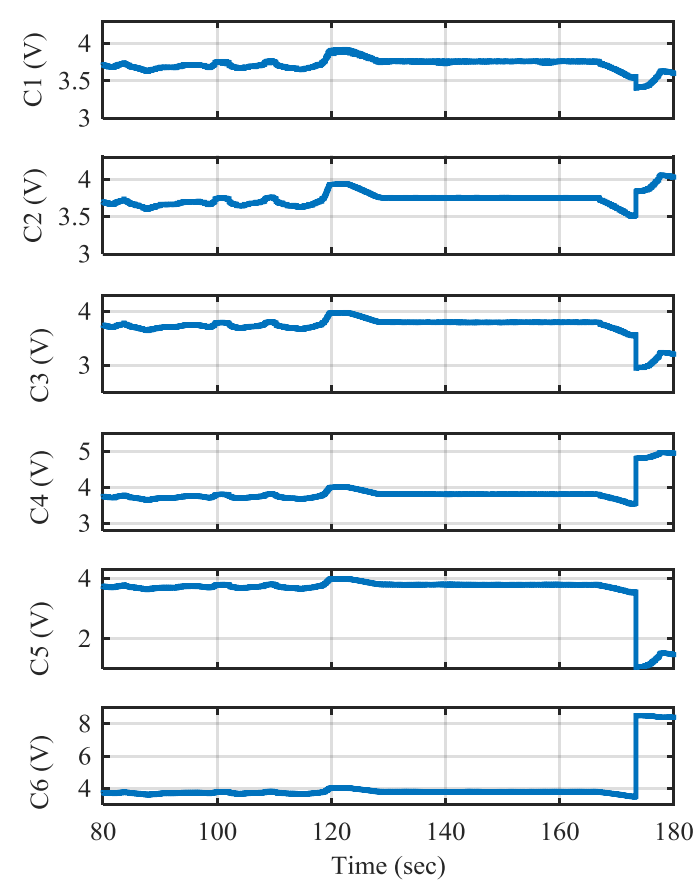

(a) Cell voltage values.
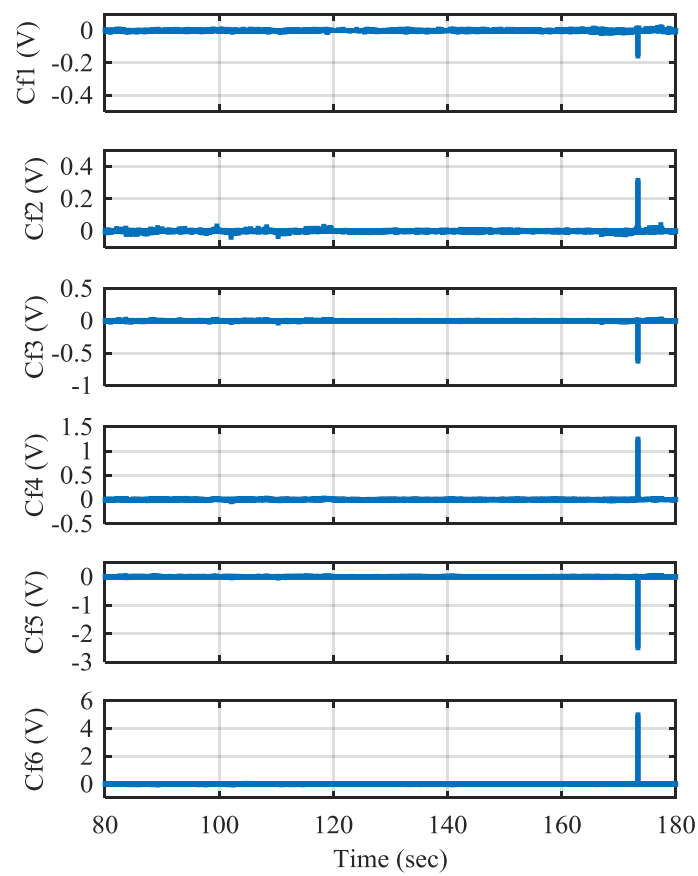

(c) Cell fault signal.
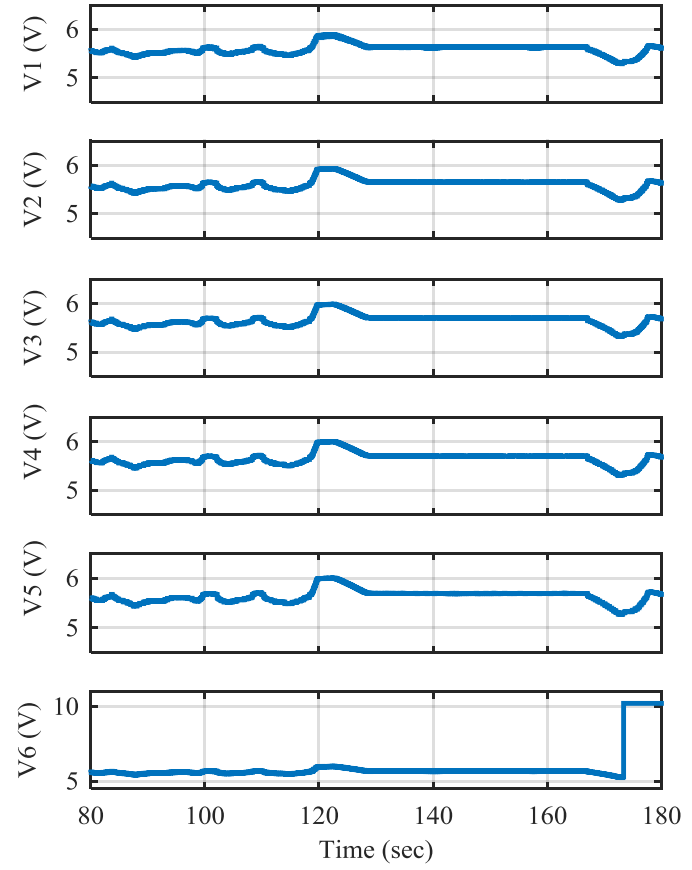

(b) Voltage measurements.
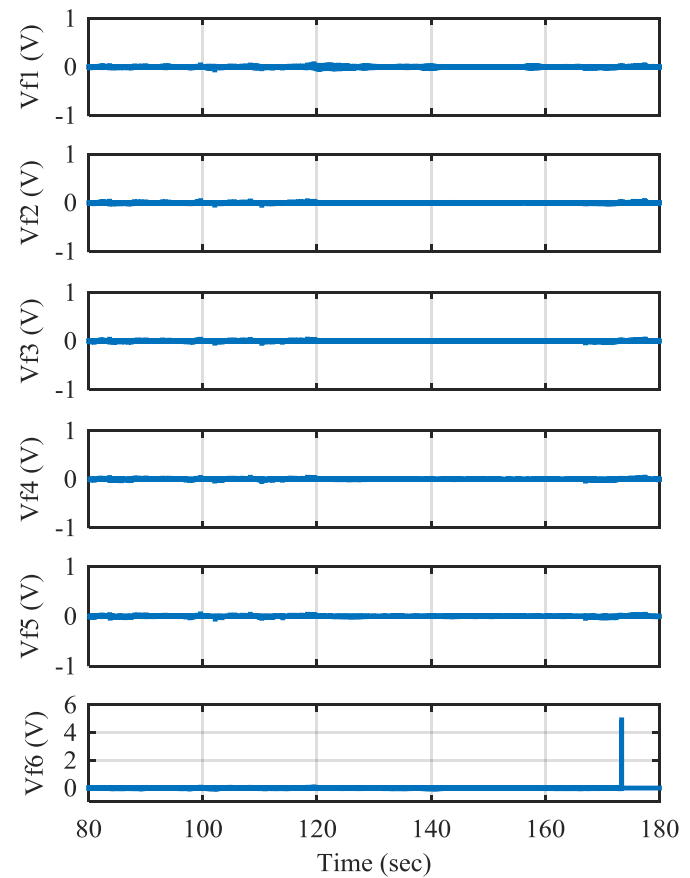

(d) Sensor fault signal.

Fig. 8 Experiment results for sensor fault diagnostic at $173.4 \mathrm{~s}$. 
As introduced in Section 2, the fault can be distinguished and isolated by the specific patterns indicated by the cell fault or sensor fault signals. Fig. 7(c) and Fig. 7(d) show that C3 is in fault condition, because 1) it is almost impossible for both V2 and V3 to be in fault condition at the same time; 2) the fault signals in Fig. 7(d) follow the specific pattern of $\left(\begin{array}{lllll}0 & 0.5 & 1 & 0 & 0\end{array}\right)^{\mathrm{T}}$, which is the third column of $\operatorname{circ}(1,0.5,0,0,0,0)$, indicating abnormal changes on $\mathrm{C} 3$.

Likewise, Fig. 8(a) and Fig. 8(b) demonstrate the sensor fault on V6 because the cell fault signals follow the pattern of $(-0.03170 .0635-0.12700 .2540-0.50791 .0159)^{\mathrm{T}}$, which is the last column of the inverse of $\operatorname{circ}(1,0.5,0,0,0,0)$. Thus, the sensor fault on V6 can be determined.

\section{Discussion}

\subsection{Benefits of the improved measurement topology}

It can be learnt from the experiment that the improved measurement topology is solvable even though the $n$ and $k$ are not prime to each other, which is not possible in the basic interleaved topology. This point is confirmed by the simulation results in Fig. 5 that the noise level gains as

$q \rightarrow 1$ for even numbers (dashed lines) approach infinity, i.e., the cell voltages cannot be solved from the sensor readings, because even $n$ numbers are not coprime to $k=2$. However, by varying the $q$ values, the noise level gains are finite and can be designed beforehand.

The other key improvement is also illustrated in Fig. 5 that the global maxima of all the curves are at $q=1$, indicating that the improved measurement topology lowers the noise level gains by varying the $q$ value.

\subsection{Selection of row entries}

The simulation and experiment demonstrate that the row entries of the measurement matrix have substantial effect on the performance of the corresponding measurement topology. The effects are discussed in detail as follows, and they are summarized in Table 1. 
First, from the cost perspective, $k$ should be as small as possible $(k \leq 2<n)$, because the number of switches increases as $k$ increases.

Second, from the invertibility perspective, the interleaved topology can be more widely applied by varying the row entries. One universal solution is the row entries in geometric series as (18). Combined with the first point, the best candidate for the first row entries is 1 and $q$ followed by all zeros $(q \neq 1)$.

After that, from the noise perspective, the maximum noise level occurs when $q=1$ for two nonzero row entries. The lower bound of the noise level gain cannot be achieved, however, an appropriate value of $q$ can be selected such that the noise level is reduced.

Next, it needs to keep in mind that $q$ should not be too small for the voltage measurements to be identified from other source of noises, and $q$ should not be too large due to its corresponding increase in the voltage level of the power supplies for the measurement circuits.

In addition, the previous work shows that the confidence level of the fault distinction increases as $k$ increases. However, it also shows that the confidence level has negligible improvement when $k$ is larger than 2 .

In summary, the optimal choice of $k$ is 2 and the two nonzero entries are 1 and $q$, where $q \neq 1$.

Table 1 Limitation and preferred choice of the first row entries of the measurement matrix.

\begin{tabular}{ll}
\hline \multicolumn{1}{c}{ Limitation } & \\
\hline Cost & Small $k$, consecutive and all '1's are preferred. \\
Invertibility of topology & Geometric series. \\
Noise & Noise can be reduced by varying $q$ (max. at $q=1)$. \\
Diagnosis confidence & Negligible improvement when $k>2$.
\end{tabular}

\section{Conclusion}


An improved non-redundancy based sensor fault isolation and detection methodology is introduced for voltage measurement in battery management systems. The basic interleaved measurement topology is able to distinguish between sensor fault and cell fault without extra sensors or cell models. Meanwhile, this basic method is constrained to the coprime relation of $n$ and $k$, which limits its engineering application. This paper provides a new proof to the coprime relation derivation, and further develops a graphical interpretation to visualize the impact of $n$ and $k$ choices to the invertibility of the measurement matrix. An improved measurement matrix is then proposed based on the graphical interpretation, which removes the coprime constraint by assigning different row entries.

Then, the hardware implementation of the improved measurement matrix is discussed. The different first row entries can be realized by simple modification of resistance values of the voltage sum circuits. It is also explained that $k=2$ is the best choice from the cost perspective.

Next, the noise level increase of the interleaved measurement method is formulated. The analytical expression of the noise level gain is derived. The results indicate that 1) the noise level of the interleaved measurement method is always larger than that of the conventional method;2) the improved method has a lower noise level than that of the basic interleaved topology.

Later discussion explains that a measurement matrix with two different row entries is the optimal choice for the interleaved measurement topology, based on the analysis in cost, invertibility, noise and detection confidence. At last, the simulation results show the same trend and limit as those in the noise analysis, and the experiment results demonstrate the broader application with a case of non-coprime $n$ and $k$ pair.

The advantages of the interleaved measurement method is that it can distinguish sensor and device fault without hardware or software redundancy. The disadvantages are 1) the noise levels 
are increased and 2) the cost in hardware implementation is increased. This paper focuses on the improvement to the basic interleaved voltage measurement method. More detailed discussion of the advantages/disadvantages of the interleaved measurement method and comparison with the existing measurement methods can be found in [19].

It is important to note that the improved interleaved measurement is not limited to battery voltage measurements. It is a methodology that can be extended to other quantity measurements, where the same measurement to multiple same subjects is needed and the fault tolerance is critical.

\section{Acknowledgements}

The authors would like to thank Dr. Xin Zhao for the great discussion and help in the study. The authors would also like to acknowledge the funding support from Nanjing Golden Dragon Bus Co., Ltd., and the U.S. Department of Energy under the Graduate Automotive Technology Education Center program.

\section{Reference}

[1] C. Mi, M.A. Masrur, D.W. Gao, Hybrid electric vehicles: principles and applications with practical perspectives, John Wiley \& Sons, 2011.

[2] T. Mahlia, T. Saktisahdan, A. Jannifar, M. Hasan, H. Matseelar, Renewable and Sustainable Energy Reviews, 33 (2014) 532-545.

[3] N. Williard, W. He, C. Hendricks, M. Pecht, Energies, 6 (2013) 4682-4695.

[4] X. Feng, M. Fang, X. He, M. Ouyang, L. Lu, H. Wang, M. Zhang, Journal of Power Sources, 255 (2014) 294-301.

[5] X. Feng, J. Sun, M. Ouyang, X. He, L. Lu, X. Han, M. Fang, H. Peng, Journal of Power Sources, 272 (2014) 457 467.

[6] J. Anderson, F. Larsson, P. Andersson, B.-E. Mellander, in: Fires in vehicles (FIVE) 2014 Conference proceedings, 2014, pp. 267-270.

[7] L. Lu, X. Han, J. Li, J. Hua, M. Ouyang, Journal of Power Sources, 226 (2013) 272-288.

[8] Y. Shang, C. Zhang, N. Cui, J.M. Guerrero, IEEE Transactions on Power Electronics, 30 (2015) 3731-3747.

[9] D. Andrea, Battery management systems for large lithium-ion battery packs, Artech house, 2010. 
[10] G.-H. Kim, K. Smith, J. Ireland, A. Pesaran, Journal of Power Sources, 210 (2012) 243-253.

[11] B. Xia, Z. Chen, C. Mi, B. Robert, in: Transportation Electrification Conference and Expo (ITEC), 2014 IEEE, IEEE, 2014, pp. 1-7.

[12] B. Xia, C. Mi, Z. Chen, B. Robert, in: Transportation Electrification Conference and Expo (ITEC), 2015 IEEE, IEEE, 2015, pp. 1-7.

[13] I. Hwang, S. Kim, Y. Kim, C.E. Seah, IEEE Transactions on Control Systems Technology, 18 (2010) $636-653$.

[14] V.P. Nelson, Computer, 23 (1990) 19-25.

[15] J. Blesa, D. Rotondo, V. Puig, F. Nejjari, Control Engineering Practice, 24 (2014) 138-155.

[16] Z. Gao, C. Cecati, S.X. Ding, IEEE Transactions on Industrial Electronics, 62 (2015) 3757-3767.

[17] A. Singh, A. Izadian, S. Anwar, Journal of Power Sources, 268 (2014) 459-468.

[18] Z. Xi, R. Jing, C. Lee, in: ASME 2015 International Design Engineering Technical Conferences and Computers and Information in Engineering Conference, American Society of Mechanical Engineers, 2015, pp. V02AT03A038-V002AT003A038.

[19] B. Xia, C. Mi, Journal of Power Sources, 308 (2016) 83-96.

[20] G. Strang, (2011).

[21] D. Geller, I. Kra, S. Popescu, S. Simanca, Preprint, Stony Brook University, (2004).

[22] I. Kra, S.R. Simanca, Notices of the AMS, 59 (2012) 368-377.

[23] C.-H. Kim, M.-Y. Kim, G.-W. Moon, IEEE Transactions on Power Electronics, 28 (2013) 3779-3787.

[24] D. Bozkurt, arXiv preprint arXiv:1202.1068, (2012).

[25] L. Fuyong, Applied Mathematics and Computation, 217 (2011) 8495-8503. 